\title{
Factors Influencing Academic Achievement in Preclinical Students
}

\author{
Yanint Raksadawan ${ }^{1}$, Chantacha Sitticharoon ${ }^{1}$, Nipith Charoenngam² ${ }^{2}$ Pailin Maikaew ${ }^{1}$, Issarawan \\ Keadkraichaiwat ${ }^{1}$ \\ ${ }^{1}$ Department of Physiology, Faculty of Medicine Siriraj Hospital, Mahidol University, Bangkok, Thailand \\ ${ }^{2}$ Department of Internal Medicine, Faculty of Medicine Siriraj Hospital, Mahidol University, Bangkok, Thailand \\ Correspondence: Chantacha Sitticharoon, Department of Physiology, Faculty of Medicine Siriraj Hospital, 2 \\ Wanglang Rd., Siriraj, Bangkok Noi, Bangkok, Thailand, 10700. Tel: 662-4199-721. Fax: 662-411-5009. E-mail: \\ chantacha.sit@mahidol.ac.th
}

Received: August 17, 2020; Accepted: September 15, 2020; Published: September 17, 2020

\begin{abstract}
Academic achievement is a major concern of preclinical students. This study aimed to determine factors influencing academic achievement in preclinical years. Questionnaires were sent to all students of the 2019 class at the $1^{\text {st }}(2019 / 1)$ and $2^{\text {nd }}$ preclinical years $(2019 / 2)$ and the 2018 class at the $2^{\text {nd }}$ preclinical year $(2018 / 2)$ without sampling, with $85.11 \%(280 / 328), 86.32 \%$ (284/328), and 83.54\% (274/328) being returned, respectively. Students were divided into Q1 (lowest)-Q2-Q3-Q4 (highest) according to quartiles of their GPA. Q4 students had higher examination expectation and achievement of study targets in all classes and more time spent on non-recorded-electure study in the 2018/2 and 2019/2 classes; but lower time spent on recorded-e-lecture study in the 2019/1 class and non-academic-internet use in the 2019/2 and 2018/2 classes than other groups. In contrast, Q1 students had higher instances of lateness and absence/year in the 2019/2 and 2018/2 classes but lower happiness scores in the 2018/2 class compared to other groups. Obese and overweight students had significantly lower GPA than normal weight and underweight students of the 2019/2 class ( $\mathrm{p}<0.05$ all). The current year GPA had significant positive correlations with the previous year GPA, examination expectation, achievement of study targets, and time spent on non-recorded-e-lecture study; but had significant negative correlations with time spent on recorded-e-lecture study and non-academic internet use, instances of lateness and absence/year, and BMI ( $p<0.05$ all). In conclusion, students with more self-regulation strategies in many aspects of self-regulated learning, including time management, learning strategies, emotion control, motivation, and self-efficacy, had better academic outcomes.
\end{abstract}

Keyword: academic achievement, preclinical students, GPA, SRL, time management

\section{Introduction}

Medical students face a lot of stressors in medical school mostly from their academic workload and conflicts from work-life imbalance (Hill, Goicochea, \& Merlo, 2018). During their preclinical years, students develop the basic knowledge needed for their clinical studies, and simultaneously adapt themselves to new medical environments including medical terminology, new subjects, and the medical thinking process (Sitticharoon, Srisuma, Kanavitoon, \& Summachiwakij, 2014).

Previous studies showed that many factors are attributed to academic success in medical school including physical factors (Al-Drees et al., 2016; Chung, Abdulrahman, Khan, Sathik, \& Rashid, 2018; Suraya, Meo, Almubarak, \& Alqaseem, 2017), self-regulated learning (SRL) (Artino et al., 2012; Cleary \& Sandars, 2011; Kötter, Wagner, Brüheim, \& Voltmer, 2017; Sobral, 2001; Song, Kalet, \& Plass, 2011; Turan \& Konan, 2012), and psychological factors (Kötter et al., 2017; Lertwilaiwittaya, Sitticharoon, Maikaew, \& Keadkraichaiwat, 2019; Sohail, 2013; Stewart, Lam, Betson, Wong, \& Wong, 1999). For physical factors, obesity has been shown to have a negative effect on study outcomes (Al-Drees et al., 2016; Suraya et al., 2017) while physical activity has a positive effect (Al-Drees et al., 2016; Chung et al., 2018).

SRL, the important strategy for life-long medical professional advancement, has a positive influence on academic achievement (Artino et al., 2012; Cleary \& Sandars, 2011; Kötter et al., 2017; Sobral, 2001; Song et al., 2011; Turan \& Konan, 2012). In general, SRL is defined as summation of affection, cognition, and behavior processes utilized to reach a set goal (van Houten-Schat et al., 2018). There are 3 strategies in SRL including 1) regulatory instruments for SRL initiation (goal setting), 2) regulatory mechanisms (planning, monitoring, learning strategies, 
motivation, and emotion control), and 3) self-appraisal (self-evaluation, self-attribution, and self-efficacy) (Sitzmann \& Ely, 2011; van Houten-Schat et al., 2018).

For the regulatory instruments, previous studies showed that highly expected examination score was positively associated with academic outcomes (Elliot, McGregor, \& Gable, 1999; Lertwilaiwittaya et al., 2019; Sitticharoon et al., 2014). For the regulatory mechanisms, high motivation to study medicine (Frischenschlager, Haidinger, \& Mitterauer, 2005; Kusurkar, Ten Cate, van Asperen, \& Croiset, 2011; Pinyopornpanish et al., 2004; Stegers-Jager, Cohen-Schotanus, \& Themmen, 2012), high percentage of study target achievement (Lertwilaiwittaya et al., 2019), regular lesson review (Lertwilaiwittaya et al., 2019; Lumley, Ward, Roberts, \& Mann, 2015), and time spent on academic-internet use (Eisen et al., 2015; S. Y. Kim, Kim, Park, Kim, \& Choi, 2017; Lertwilaiwittaya et al., 2019) had a positive effect on study outcomes, while time spent on non-academic internet use (AlFaris et al., 2018; Azizi, Soroush, \& Khatony, 2019; Lertwilaiwittaya et al., 2019) and recorded-e-lecture study (Eisen et al., 2015; S. Y. Kim et al., 2017; Lertwilaiwittaya et al., 2019) had a negative effect. Class attendance has inconsistent results. Some studies showed that increased instances of lateness and/or absence had a negative association with academic outcomes (Liles, Vuk, \& Tariq, 2018; Sitticharoon et al., 2014); however, some studies showed no association (Eisen et al., 2015; Kauffman, Derazin, Asmar, \& Kibble, 2018). For psychological factors, stress has a negative (Kötter et al., 2017; Lertwilaiwittaya et al., 2019; Sohail, 2013; Stewart et al., 1999) while happiness has a positive effect on academic performance (Chen \& Lu, 2009; Datu, Valdez, Cabrera, \& Salanga, 2017; Kötter et al., 2017; Lertwilaiwittaya et al., 2019; Sohail, 2013; Stewart et al., 1999).

The aim of this study was to determine factors associated with academic success in the students of class of 2019 in the $1^{\text {st }}(2019 / 1)$ and $2^{\text {nd }}(2019 / 2)$ preclinical years and the students of class of 2018 in the $2^{\text {nd }}$ preclinical year (2018/2) by 1) comparisons of GPA between groups of BMI and motivation to study medicine, 2) comparisons of many factors among groups of quartiles of students' GPA, 3) determining correlations between GPA and other factors, and 4) determining factors which contributed to GPA of students using regression analysis. By doing the study in many classes, identification of factors related to academic achievement could become apparent. By revelation of factors related to academic outcomes, we would be able to provide more efficient guidance for students who would like to improve their academic achievement in their preclinical years, early detection strategies for detrimental study habits, and early prevention of adverse outcomes.

\section{Materials and Methods}

\subsection{Study Protocol and Data Collection}

Prior to data collection, the study's protocol was approved by the Siriraj Institutional Review Board (769/2557(EC3)) and we obtained informed consents from all subjects.

\subsection{Research Design}

This is a cross sectional questionnaire-based study. The questionnaires were sent out to all preclinical students of 2018 and 2019 classes without sampling. We gathered data from 3 periods, at the end of the 2019/1, 2019/2, and $2018 / 2$ classes. There were $85.11 \%$ (280/329), 86.32\% (284/329), and 83.54\% (274/328) questionnaire respondents for the 2019/1, 2019/2, and 2018/2 classes, respectively. The respondents were comprised of 57.8\% males and $42.2 \%$ females in the $2019 / 1$ class, $58.3 \%$ males and $41.7 \%$ females in the $2019 / 2$ class, and $51.4 \%$ males and $48.6 \%$ females in the 2018/2 class. The age range was between 19-21 years for the 2019/1 students and 20-22 years for the 2019/2 and 2018/2 students.

\subsection{Type of Curriculum and Course Setup}

Thai students enter medical school after high school graduation. The Doctor of Medicine program, which is a 6year curriculum, at the Faculty of Medicine Siriraj Hospital, Mahidol University, Thailand, is divided into 3 parts, the premedical, preclinical, and clinical parts. The $1^{\text {st }}$ year is the premedical year, where students study general knowledge including basic sciences, social sciences, and languages. For the $2^{\text {nd }}$ year, or the $1^{\text {st }}$ preclinical year, students study biochemistry, histology, embryology, gross anatomy, neuroanatomy, physiology, and minor subjects. For the $3^{\text {rd }}$ year, or the $2^{\text {nd }}$ preclinical year, students study pharmacology, pathology, immunology, microbiology, parasitology, clinical pathology, and minor subjects. The $4^{\text {th }}$ to $6^{\text {th }}$ years are clinical years.

\subsection{Questionnaire}

The Thai-language self-reported questionnaire was firstly assessed by medical students to confirm its clarity and readability. It was then submitted to a grant committee for review, validation, and approval for readability, comprehensiveness, rational analysis, and clarity. The calculated internal consistency (reliability) of data by the Cronbach's alpha method was 0.886 . The questionnaire was comprised of 3 parts including box-ticking questions, open-ended questions, and happiness scale. 
Box-ticking questions included students' motivation to study medicine (low, medium, or high), and their stress level $(1=$ very low, $2=$ low, $3=$ medium, $4=$ high, and $5=$ very high $)$. The open-ended questions included questions regarding their demographic data, their study behaviors, and their wake-up time (hours). Demographic questions asked for data including body weight $(\mathrm{kg})$ and height $(\mathrm{cm})$ to obtain body mass index (BMI) which was categorized into 4 groups including underweight $\left(<18.5 \mathrm{~kg} / \mathrm{m}^{2}\right)$, normal weight $\left(18.5-22.9 \mathrm{~kg} / \mathrm{m}^{2}\right)$, overweight (23$\left.24.9 \mathrm{~kg} / \mathrm{m}^{2}\right)$, and obese $\left(>25 \mathrm{~kg} / \mathrm{m}^{2}\right)$ according to the BMI classification of Asian populations ("Appropriate bodymass index for Asian populations and its implications for policy and intervention strategies," 2004). Study behavior questions asked about time spent on internet use for non-academic and academic purposes (hour(s)/day), total time spent on lesson review (hour(s)/day), time spent on recorded-e-lecture study (hour(s)/week), time spent on nonrecorded-e-lecture study (hour(s)/week), the students' examination expectation score in percentage, and a percentage of achievement of study targets. Recorded-e-lecture was defined as video records of a class lecture which was uploaded onto the internet where students could review the corresponding lecture at any time after the class. The examination expectation score in percentage was obtained by the students' prediction of the score percentage they expected to get. The percentage of achievement of study targets means the percentage of content they studied in comparison to the total content they expected to study.

The happiness score was acquired according to the Fordyce Emotions Questionnaire method (Rabito-Alcon \& Rodriguez-Molina, 2016). Students were asked to rate their happiness by scoring from 0 (extremely unhappy) to 10 (extremely happy).

\subsection{Academic Achievement and Class Attendance}

Academic achievement, represented as GPA, and class attendance, represented as instances of lateness and absence per academic year, were obtained from the undergraduate education department. Class attendance, lateness to class, and absence from class, were monitored by fingerprint scanning at the beginning of the first class of each day.

\subsection{Subgroup Analysis}

Subgroup analysis was done according to groups of different classes, BMI, motivation to study medicine, and GPA quartiles of students with the Q1 being the quartile with the lowest GPA and the Q4 being the quartile with the highest GPA. The factors that were evaluated included examination expectation score in percentage, the percentage of achievement of study targets, time spent on lesson review, time spent on recorded-e-lecture study, time spent on non-recorded-e-lecture study, time spent on non-academic internet use, time spent on academic internet use, instances of absence and lateness per year, happiness of students, and stress level.

\subsection{Statistical Analysis}

For statistical analysis, Statistical Package for Social Science version 18 was used. Normality was tested using the Kolmogorov-Smirnov test. Comparisons of data with more than 2 groups were done using one-way analysis of variance (ANOVA). Post hoc analyses were followed, using the Fisher's least significant difference test where appropriate. For non-normally distributed and ordinal variables, the non-parametric test was used. To determine correlations between 2 factors, parametric parameters used the Pearson product-moment correlation coefficient while non-parametric parameters used the Spearman's rank correlation coefficient. To test which factors had a significant contribution to the GPA of students in each academic year, multiple linear regression analysis was used. A p-value less than 0.05 is considered statistical significance.

\section{Results}

\subsection{Motivation and Stress Levels}

Students reported low, medium, and high motivation to study medicine for $3.8 \%, 34.4 \%$, and $61.9 \%$, respectively for the $2019 / 1$ class; $3.9 \%, 38.7 \%$, and $57.4 \%$, respectively for the $2019 / 2$ class; and $1.5 \%, 32.5 \%$, and $66.1 \%$, respectively for the $2018 / 2$ class. Students reported very low, low, medium, high, and very high stress levels for $4.8 \%, 15.8 \%, 48.1 \%, 27.1 \%$, and $4.1 \%$, respectively for the $2019 / 1$ class; $5.9 \%, 17.0 \%, 53.1 \%, 19.7 \%$, and $4.3 \%$, respectively for the $2019 / 2$ class; and $5.2 \%, 14.4 \%, 52.2 \%, 24.1 \%$, and $4.1 \%$, respectively for the $2018 / 2$ class. 
A

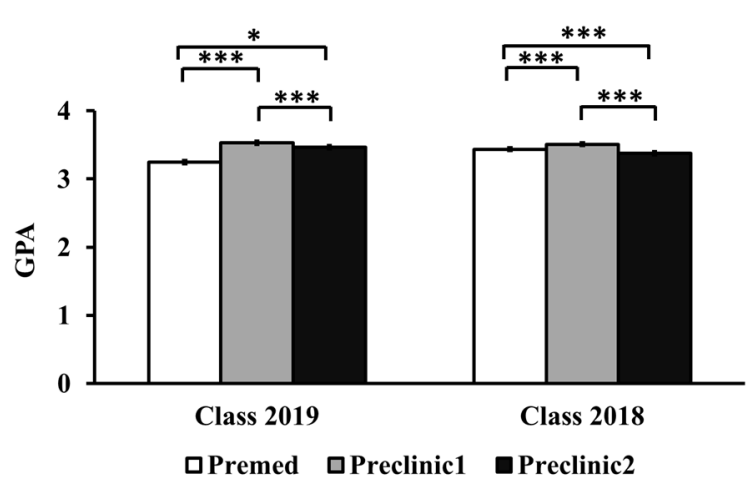
Cotivation to study medicine
of students of the 2019 class

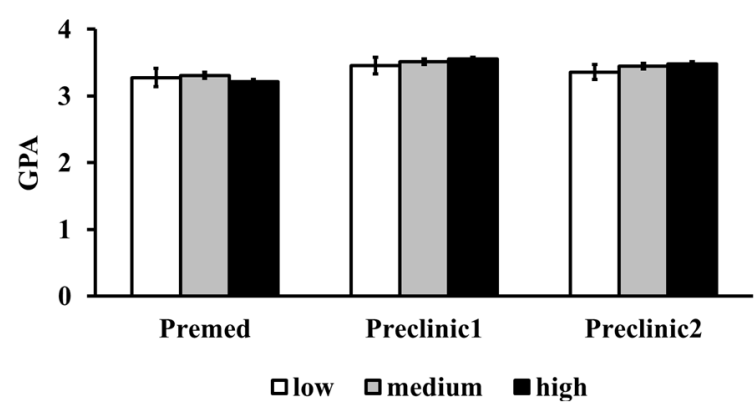

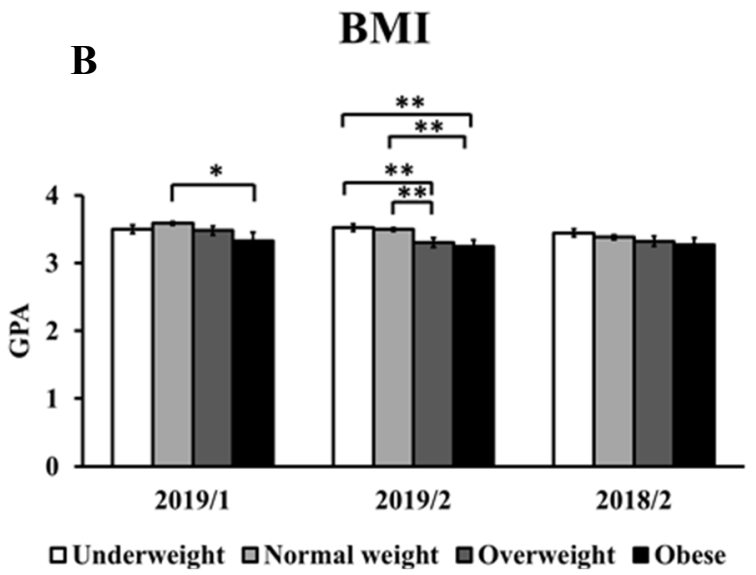

\section{Motivation to study medicine of students of the 2018 class}

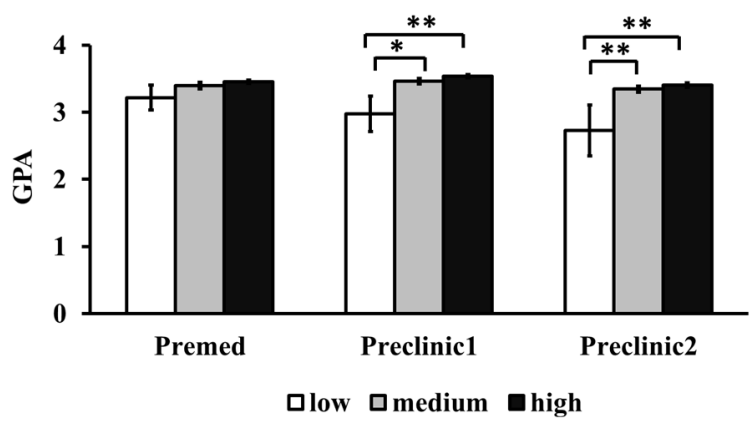

Figure 1.

Grade point average (GPA) of students compared between groups of academic year (A), body mass index (BMI) (B), and motivation to study medicine of the class of 2019 (C) and 2018 (D). Premed represents the premedical year, Preclinic1 and Preclinic2 represent the $1^{\text {st }}$ and $2^{\text {nd }}$ preclinical years, respectively. 2019/1 and 2019/2 represent the class of 2019 in the $1^{\text {st }}$ and $2^{\text {nd }}$ preclinical years, respectively. 2018/2 represents the class of 2018 in the $2^{\text {nd }}$ preclinical year.

\subsection{GPA of Students Compared between Groups of Academic Year, BMI, and Motivation to Study Medicine}

Comparisons of GPA according to class and academic year, BMI, and motivation to study medicine are shown in Figure 1.

Data are shown as mean (standard error of the mean, S.E.M.), $* \mathrm{p}<0.05 * * \mathrm{p}<0.01$, $* * * \mathrm{p}<0.001$ compared between groups

Students of class of 2018 and 2019 had the highest GPA in the first preclinical year which was significantly higher than the second preclinical year and the premedical year ( $p<0.001$ all) (Figure 1A). Furthermore, GPA of students in the second preclinical year was significantly higher $(p<0.05)$ in the class of 2019 , while it was lower in the class of $2018(\mathrm{p}<0.001)$ compared to the premedical year (Figure 1A).

For different BMI groups, obese students of the 2019/1 class had significantly lower GPA than normal weight students $(\mathrm{p}<0.05)$ (Figure 1B). In the 2019/2 class, overweight and obese students had significantly lower GPA than underweight and normal weight students ( $\mathrm{p}<0.01$ all) (Figure 1B). In the 2018/2 class, GPA was comparable between different BMI groups (Figure 1B).

For motivation to study medicine, GPA of students of the 2019 class was comparable between different motivation levels for all academic years (Figure 1C). However, for the 2018 class, students with low motivation level had significantly lower GPA compared to students with medium or high motivation in both first and second preclinical years $(\mathrm{p}<0.05)($ Figure 1D). 


\section{A Examination expectation score}

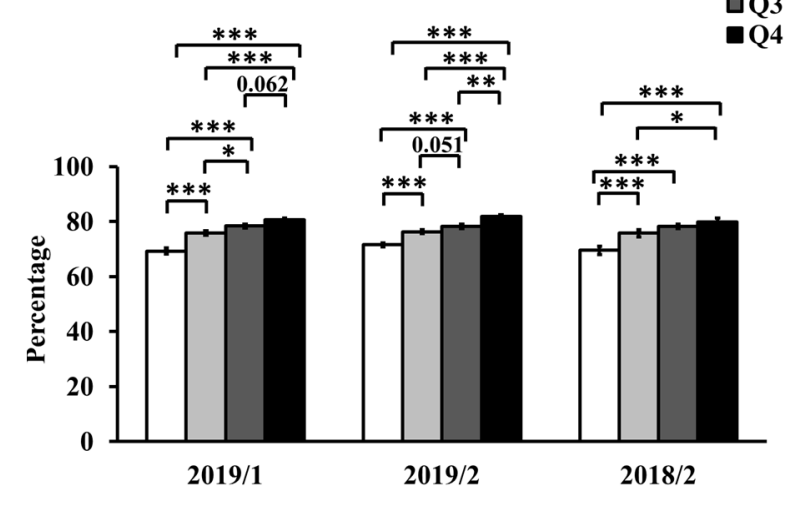

C Time spent on lesson review 口Q1
व2 口Q3 -Q4

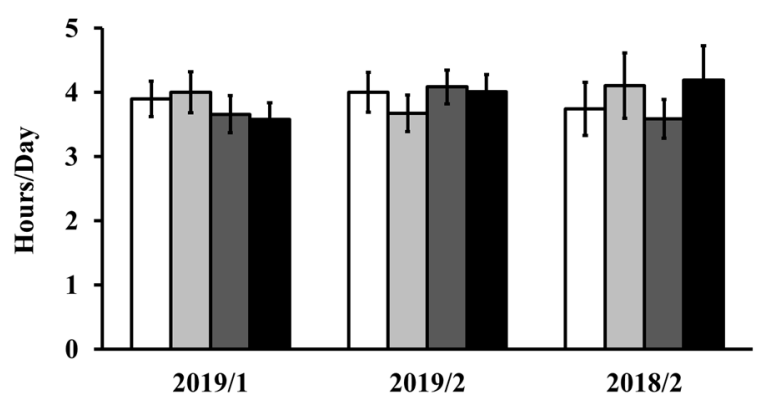

$\begin{array}{lll}\text { E Time spent on non-recorded- } & \text { QQ1 } \\ \text { QQ2 }\end{array}$ e-lecture study QQ3

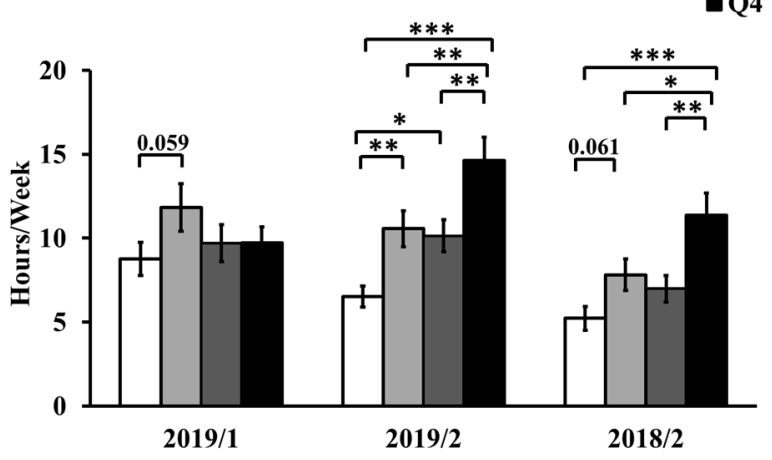

B Acheivement of study targets

वQ1

口Q3 -Q4

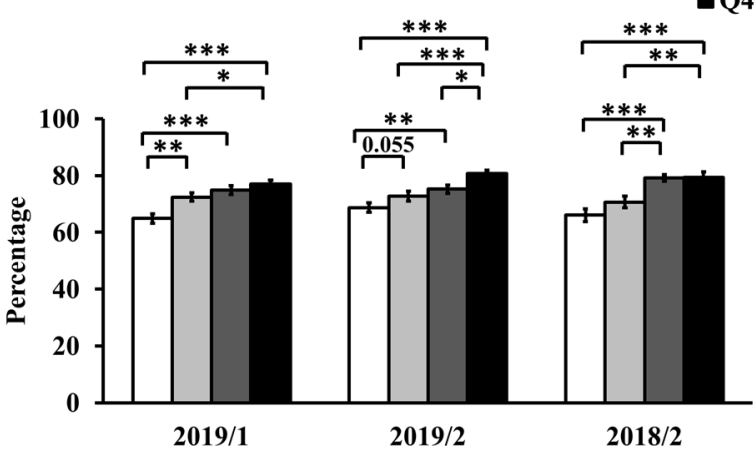

$\begin{array}{lll}\text { D Time spent on recorded- } & \square \mathrm{Q} 1 \\ \mathrm{QQ2}\end{array}$ e-lecture study

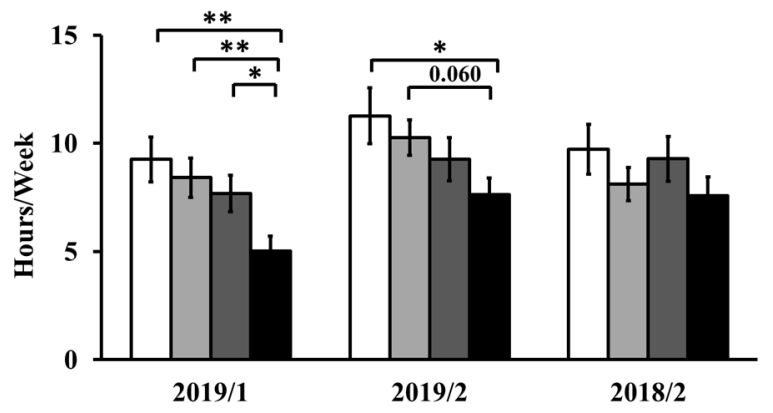

F Time spent on academic internet use

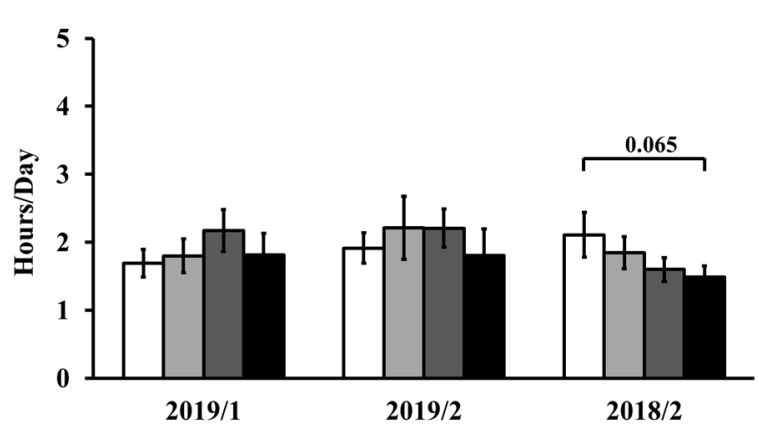



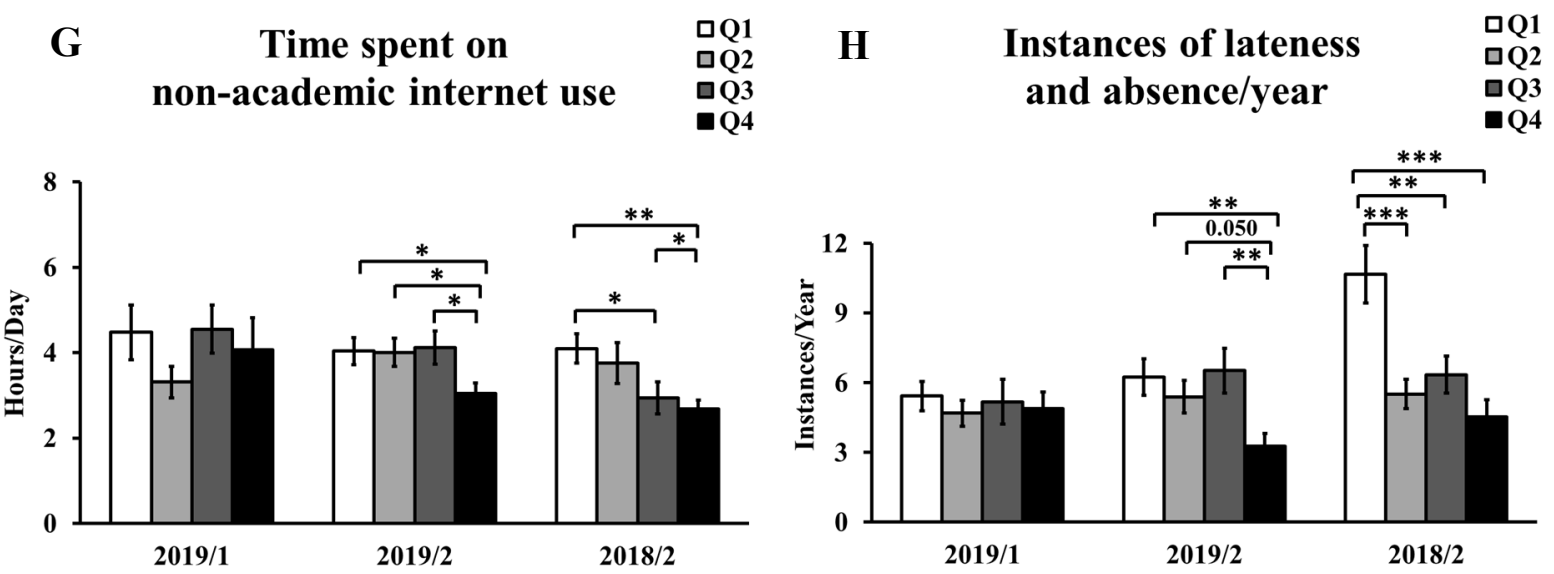

\section{Happiness of students

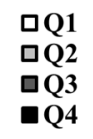

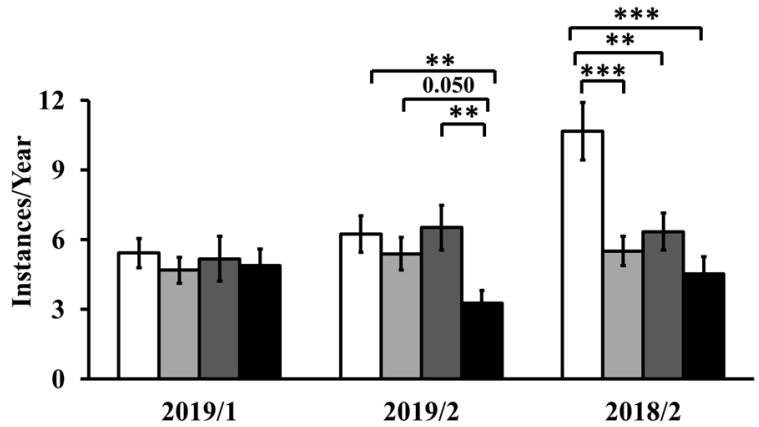
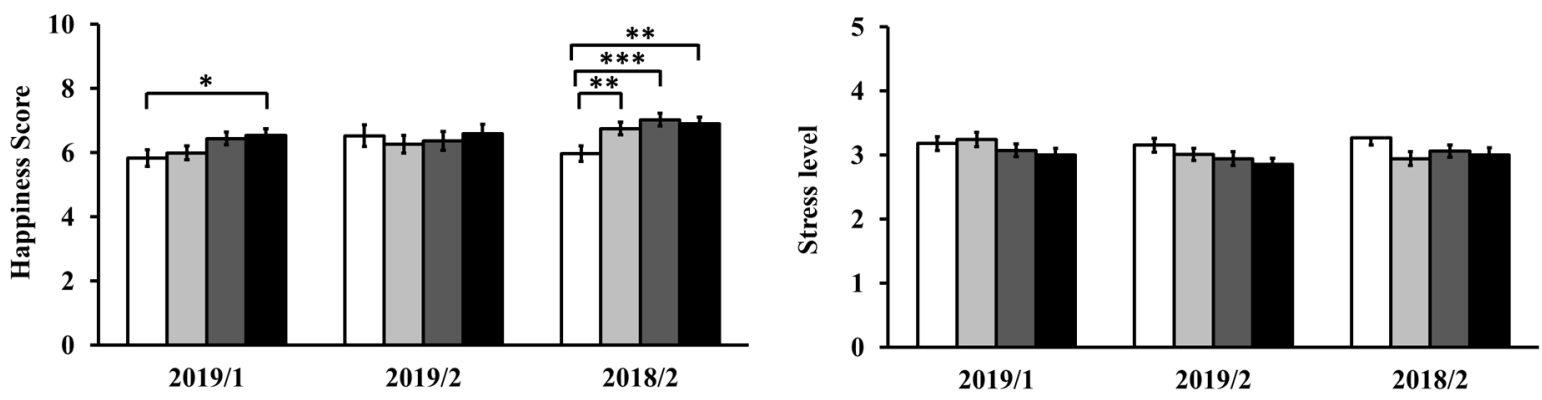

Figure 2.

Comparisons of factors between groups of quartiles of grade point average (GPA) of students including examination expectation score in percentage (A), the percentage of achievement of study targets (B), time spent on lesson review (C), time spent on recorded-e-lecture study (D), time spent on non-recorded-e-lecture study (E), time spent on academic internet use (F), time spent on non-academic internet use (G), instances of lateness and absence/year (H), happiness of students (I), and stress level (J).

$2019 / 1$ and 2019/2 represent the class of 2019 in the $1^{\text {st }}$ and $2^{\text {nd }}$ preclinical years, respectively. 2018/2 represents the class of 2018 in the $2 \mathrm{nd}$ preclinical year. Data are shown as mean (standard error of the mean, S.E.M.), * $\mathrm{p}<0.05$, $* * \mathrm{p}<0.01, * * * \mathrm{p}<0.001$ compared between groups.

\subsection{Comparisons of Factors between Groups of Quartiles of GPA of Students}

Comparisons of factors between groups of quartiles of GPA are demonstrated in Figure 2.

Examination expectation in percentage in all classes was highest in the Q4 group, which was significantly higher compared to the Q1 and Q2 groups; was significantly higher in the Q3 group compared to the Q1 group; and was significantly higher in the $\mathrm{Q} 2$ group compared to the $\mathrm{Q} 1$ group ( $<0.05$ all) (Figure $2 \mathrm{~A}$ ).

The percentage of achievement of study targets in all classes was highest in the Q4 group, which was significantly higher than the Q1 and Q2 groups; and was significantly higher in the Q3 group than the Q1 group ( $<0.05$ all) (Figure 2B).

Time spent on lesson review in all groups was comparable in all classes (Figure 2C). However, the Q4 group had significantly lower time spent on recorded-e-lecture study than the Q1, Q2, and Q3 groups in the 2019/1 class; and than the Q1 group in the 2019/2 class ( $p<0.05$ all) (Figure 2D). In contrast, Q4 students had significantly higher time spent on non-recorded-e-lecture study than Q1, Q2, and Q3 students for the 2019/2 and 2018/2 classes, and that of Q2 and Q3 students was significantly higher than Q1 students of the 2019/2 class ( $p<0.05$ all) (Figure 2E). 
Time spent on academic internet use was comparable between groups in all 3 classes (Figure 2F). However, time spent on non-academic internet use of the Q4 group was significantly lower than the Q1, Q2, and Q3 groups in the 2019/2 class; and than the Q1 and Q3 groups in the 2018/2 class ( $\mathrm{p}<0.05$ all) (Figure 2G). The Q3 group of the 2018/2 class spent significantly less time on non-academic-internet use than the Q1 group ( $p<0.05)$ (Figure 2G).

Instances of lateness and absence per year were significantly lower in the Q4 students than the Q1 and Q3 students $(\mathrm{p}<0.05)$ and tended to be lower in the Q4 students than the Q2 students of the 2019/2 class; and was significantly higher in the Q1 students than the Q2, Q3, and Q4 students of the 2018/2 class ( $\mathrm{p}<0.05$ all) (Figure 2H).

Happiness of the Q1 students was significantly lower than the Q4 students of the 2019/1 class; and than the Q2, Q3, and Q4 students of the 2018/2 class ( $\mathrm{p}<0.05$ all) (Figure 2I). Stress level was comparable in all quartiles for all classes (Figure 2J).

\subsection{Correlations between Students' GPA and other Factors}

Correlations between students' GPA and other factors are shown in Table 1.

Table 1. Correlations between students' GPA and other factors. 2019/1 and 2019/2 represent the class of 2019 in the $1^{\text {st }}$ and $2^{\text {nd }}$ preclinical years, respectively.

\begin{tabular}{|c|c|c|c|c|}
\hline \multicolumn{2}{|c|}{ GPA of students in the $1^{\text {st }}$ preclinical year } & \multicolumn{3}{|c|}{ GPA of students in the $2^{\text {nd }}$ preclinical year } \\
\hline Factors & $\mathbf{R}$ & Factors & $\mathbf{R}$ & $\mathbf{R}$ \\
\hline Class & 2019/1 & Class & 2019/2 & 2018/2 \\
\hline \multirow{10}{*}{$\begin{array}{l}\text { - Premedical year GPA } \\
\text { - Examination expectation } \\
\text { score in percentage } \\
\text { - Percentage of achievement of } \\
\text { study targets } \\
\text { - Time spent on recorded-e- } \\
\text { lecture study (hours/week) }\end{array}$} & $0.658 * * *$ & - Premedical year GPA & $0.547 * * *$ & $0.497 * * *$ \\
\hline & $0.493 * * *$ & $-1^{\text {st }}$ preclinical year GPA & $0.857 * * *$ & $0.854 * * *$ \\
\hline & $0.354 * * *$ & $\begin{array}{l}\text { - Examination expectation score in } \\
\text { percentage }\end{array}$ & $0.532 * * *$ & $0.405 * * *$ \\
\hline & $-0.194 * *$ & $\begin{array}{l}\text { - Percentage of achievement of } \\
\text { study targets }\end{array}$ & $0.296^{* * *}$ & $0.366^{* * *}$ \\
\hline & & $\begin{array}{l}\text { - Time spent on non-recorded-e- } \\
\text { lecture study (hours/week) }\end{array}$ & $0.268 * * *$ & $0.226 * * *$ \\
\hline & & $\begin{array}{l}\text { - Time spent on internet for non- } \\
\text { academic use (hours/day) }\end{array}$ & -0.086 & $-0.213 * * *$ \\
\hline & & $\begin{array}{l}\text { - Instances of lateness and } \\
\text { absence/year }\end{array}$ & -0.089 & $-0.349 * * *$ \\
\hline & & $\begin{array}{l}\text { - Time spent on recorded-e-lecture } \\
\text { study (hours/week) }\end{array}$ & $-0.143 * * *$ & -0.072 \\
\hline & & - Instances of absence per year & $-0.129 *$ & $-0.304 * * *$ \\
\hline & & - BMI & $-0.214 * * *$ & $\begin{array}{l}-0.107 \\
(p=0.078)\end{array}$ \\
\hline
\end{tabular}

2018/2 represents the class of 2018 in the $2^{\text {nd }}$ preclinical year. GPA $=$ grade point average, $\mathrm{R}=$ correlation coefficient, BMI $=$ body mass index, ${ }^{*} \mathrm{p}<0.05,{ }^{*} \mathrm{p}<0.01,{ }^{* * *} \mathrm{p}<0.001$.

GPA of the 2019/1 students had significant positive correlations with premedical year GPA $(\mathrm{R}=0.658)$, examination expectation score in percentage $(R=0.493)$, the percentage of achievement of study targets $(R=0.354)$, but had a significant negative correlation with time spent on recorded-e-lecture study $(\mathrm{R}=-0.194)(\mathrm{p}<0.01$ all $)$ (Table 1).

GPA of the 2019/2 students was significantly positively correlated with premedical year GPA ( $R=0.547)$, the $1^{\text {st }}$ preclinical year GPA $(R=0.857)$, examination expectation score in percentage $(R=0.532)$, the percentage of achievement of study targets $(\mathrm{R}=0.296)$, time spent on non-recorded-e-lecture study $(\mathrm{R}=0.268)$; but was significantly negatively correlated with time spent on recorded-e-lecture study $(\mathrm{R}=-0.143)$, instances of absence per year $(\mathrm{R}=-0.129)$, and BMI $(\mathrm{R}=-0.214)(\mathrm{p}<0.05$ all $)($ Table 1$)$.

GPA of the 2018/2 students had significant positive correlations with premedical year GPA $(R=0.497)$, the $1^{\text {st }}$ preclinical year GPA $(R=0.854)$, examination expectation score in percentage $(R=0.405)$, the percentage of achievement of study targets $(\mathrm{R}=0.366)$, time spent on non-recorded-e-lecture study $(\mathrm{R}=0.226)$; but had significant 
negative correlations with time spent on non-academic internet use $(\mathrm{R}=-0.213)$, instances of absence per year $(\mathrm{R}=$ $-0.304)(\mathrm{p}<0.001$ all); and tended to have a negative correlation with BMI $(\mathrm{p}=0.078)$ (Table 1$)$.

\subsection{Multivariate Regression Analysis}

Multivariate regression analysis for GPA of students of the classes of 2019 and 2018 is shown in Table 2 and 3, respectively.

Table 2. Multivariate regression analysis for GPA of students of the class of 2019

\begin{tabular}{|c|c|c|c|c|c|c|c|c|c|}
\hline $\begin{array}{c}\text { Dependent } \\
\text { variable }\end{array}$ & Model & $\mathrm{R}$ & $\mathrm{R}^{2}$ & P-value & & Coefficient & $\begin{array}{c}\text { Standard } \\
\text { error }\end{array}$ & T-value & P-value \\
\hline \multirow{14}{*}{ 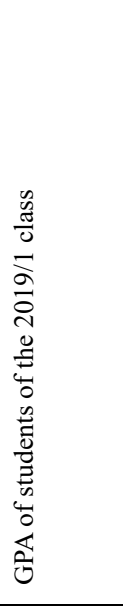 } & \multirow{2}{*}{1} & \multirow{2}{*}{0.649} & \multirow{2}{*}{0.421} & \multirow{2}{*}{$<0.001$} & Constant & 1.725 & 0.129 & 13.370 & $<0.001 * * *$ \\
\hline & & & & & Premedical year GPA & 0.558 & 0.039 & 14.200 & $<0.001 * * *$ \\
\hline & \multirow{3}{*}{2} & \multirow{3}{*}{0.708} & \multirow{3}{*}{0.501} & \multirow{3}{*}{$<0.001$} & Constant & 1.075 & 0.240 & 4.483 & $<0.001 * * *$ \\
\hline & & & & & Premedical year GPA & 0.572 & 0.039 & 14.704 & $<0.001 * * *$ \\
\hline & & & & & Wake up time (hours) & 0.09 & 0.028 & 3.195 & $0.002 * *$ \\
\hline & \multirow{4}{*}{3} & \multirow{4}{*}{0.678} & \multirow{4}{*}{0.460} & \multirow{4}{*}{$<0.001$} & Constant & 0.826 & 0.250 & 3.299 & $0.001 * *$ \\
\hline & & & & & Premedical year GPA & 0.575 & 0.038 & 14.992 & $<0.001^{* * *}$ \\
\hline & & & & & Wake up time (hours) & 0.100 & 0.028 & 3.556 & $<0.001 * * *$ \\
\hline & & & & & Happiness score & 0.028 & 0.009 & 3.011 & $0.003 * *$ \\
\hline & \multirow{5}{*}{4} & \multirow{5}{*}{0.69} & \multirow{5}{*}{0.477} & \multirow{5}{*}{$<0.001$} & Constant & 0.781 & 0.247 & 3.158 & $0.002 * *$ \\
\hline & & & & & Premedical year GPA & 0.584 & 0.038 & 15.388 & $<0.001^{* * *}$ \\
\hline & & & & & Wake up time (hours) & 0.108 & 0.028 & 3.869 & $<0.001^{* * *}$ \\
\hline & & & & & Happiness score & 0.028 & 0.009 & 3.025 & $0.003 * *$ \\
\hline & & & & & Instances of lateness/year & -0.013 & 0.004 & -2.977 & $0.003 * *$ \\
\hline \multirow{9}{*}{ 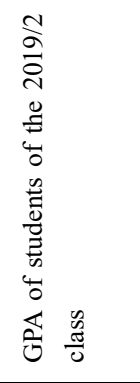 } & \multirow{2}{*}{1} & \multirow{2}{*}{0.843} & \multirow{2}{*}{0.711} & \multirow{2}{*}{$<0.001$} & Constant & 0.664 & 0.115 & 5.767 & $<0.001 * * *$ \\
\hline & & & & & $1^{\text {st }}$ preclinical year GPA & 0.792 & 0.032 & 24.531 & $<0.001 * * *$ \\
\hline & \multirow{3}{*}{2} & \multirow{3}{*}{0.863} & \multirow{3}{*}{0.745} & \multirow{3}{*}{$<0.001$} & Constant & 0.378 & 0.119 & 3.164 & $0.002 * *$ \\
\hline & & & & & $1^{\text {st }}$ preclinical year GPA & 0.824 & 0.031 & 26.676 & $<0.001 * * *$ \\
\hline & & & & & Happiness score & 0.027 & 0.005 & 5.725 & $<0.001^{* * *}$ \\
\hline & \multirow{4}{*}{3} & \multirow{4}{*}{0.867} & \multirow{4}{*}{0.751} & \multirow{4}{*}{$<0.001$} & Constant & 0.566 & 0.14 & 4.052 & $<0.001 * * *$ \\
\hline & & & & & $1^{\text {st }}$ preclinical year GPA & 0.818 & 0.031 & 26.686 & $<0.001 * * *$ \\
\hline & & & & & Happiness score & 0.027 & 0.005 & 5.606 & $<0.001 * * *$ \\
\hline & & & & & Body weight $(\mathrm{kg})$ & -0.003 & 0.001 & -2.525 & $0.012 *$ \\
\hline
\end{tabular}

2019/1 and 2019/2 represent the class of 2019 in the $1^{\text {st }}$ and $2^{\text {nd }}$ preclinical years, respectively. GPA $=$ grade point average, $\mathrm{R}=$ correlation coefficient, ${ }^{*} \mathrm{p}<0.05,{ }^{* *} \mathrm{p}<0.01,{ }^{* * *} \mathrm{p}<0.001$.

Table 3. Multivariate regression analysis for GPA of students of the class of 2018 in the $2^{\text {nd }}$ preclinical year $(2018 / 2)$.

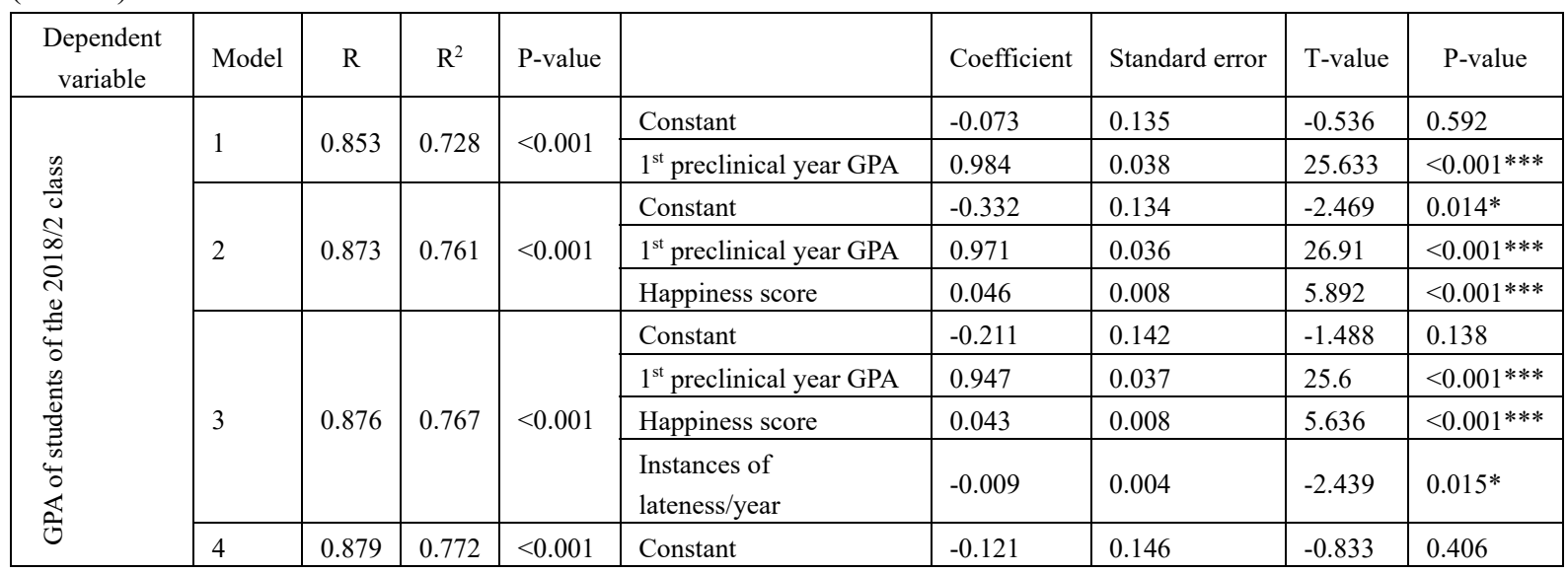




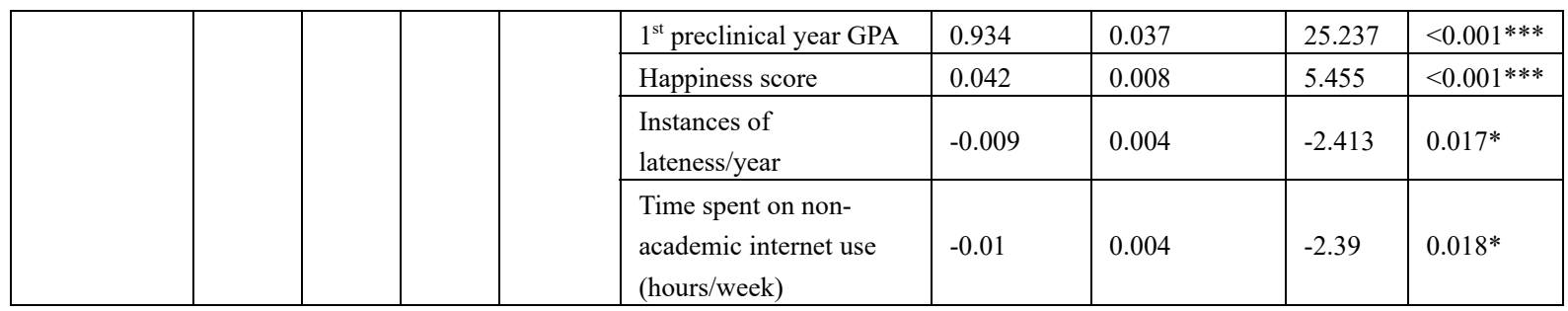

$\mathrm{GPA}=$ grade point average, $\mathrm{R}=$ correlation coefficient, ${ }^{*} \mathrm{p}<0.05, * * * \mathrm{p}<0.001$.

By setting GPA of the 2019/1 students as a dependent variable, significant interaction models were found by setting premedical year GPA (Model 1); or premedical year GPA and wake up time (Model 2); or premedical year GPA, wake up time, and happiness score (Model 3); or premedical year GPA, wake up time, happiness score, and instances of lateness per year (Model 4) as independent variables.

When setting GPA of the 2019/2 students as a dependent variable, significant interaction models were found by using either the $1^{\text {st }}$ preclinical year GPA (Model 1); or $1^{\text {st }}$ preclinical year GPA and happiness score (Model 2); or $1^{\text {st }}$ preclinical year GPA, happiness score, and body weight (Model 3) as independent variables.

When setting GPA of the 2018/2 students as a dependent variable, significant interaction models were observed by using either the $1^{\text {st }}$ preclinical year GPA (Model 1); or $1^{\text {st }}$ preclinical year GPA and happiness score (Model 2); or $1^{\text {st }}$ preclinical year GPA, happiness score, and instances of lateness per year (Model 3); or $1^{\text {st }}$ preclinical year GPA, happiness score, instances of lateness per year, and time spent on non-academic internet use (hours/week) (Model 4) as independent variables.

\section{Discussion}

This study determined the factors associated with academic achievement in 2 classes, the class of 2018 and 2019. For the class of 2019, we performed a follow-up longitudinal assessment for 2 academic years. We found that GPA of students in both classes was in the same direction, with significantly higher GPA found in the $1^{\text {st }}$ preclinical year compared to the premedical and $2^{\text {nd }}$ preclinical years. These results suggest that GPA of students in different classes had similar trends in each academic year.

In all classes, we found that higher GPA students had significantly higher examination expectation scores than lower GPA students. Furthermore, we found significant positive correlations between GPA and examination expectation scores in percentage in all classes. Our results were in agreement with previous studies revealing that students with higher exam expectation scores had higher academic outcomes in national license scores (Lertwilaiwittaya et al., 2019) and higher subject scores (Sitticharoon et al., 2014). Examination expectation is the goal set by students for their desired academic outcomes. Goal setting is a part of the regulatory instruments of SRL, which provides a target for students to initiate their learning process and adjusts their plans if there is a possibility of not reaching their goals (Sitzmann \& Ely, 2011). In the past, high target level has been shown to have positive associations with more motivation to reach the set goal and higher study performance (Vancouver \& Kendall, 2006).

The percentage of study target achievement was highest in the Q4 students in all classes compared to others. Furthermore, there was significant positive correlations between GPA and the percentage of achievement of study targets in all classes. Our results were similar with previous studies revealing that students with the higher percentage of achievement of study targets had higher examination scores (Lertwilaiwittaya et al., 2019). The percentage of achievement of study targets reflects students' effort, representing the amount of time spent on learning (Sitzmann \& Ely, 2011). Effort is a part of the regulatory mechanism of SRL and could also be adjusted when it seems like they will not reach their goal (Sitzmann \& Ely, 2011). Collectively, students with higher examination expectation might be a reflection of higher determination, probably leading to more effort put in, which might result in higher achievement of study targets leading to examination accomplishment (Lertwilaiwittaya et al., 2019).

In our study, the total time spent for study review was comparable in all quartile groups. However, the Q4 students of the 2019/1 class spent a significantly lower amount of time using recorded-e-lecture study compared to the Q1, Q2, and Q3 students. On the other hand, the Q4 students of the 2019/2 and 2018/2 classes spent a significantly higher amount of time with non-recorded-e-lecture study than the Q1, Q2, and Q3 students. Furthermore, GPA had positive correlations with time spent on non-recorded-e-lecture study in the 2019/2 and 2018/2 classes, but had negative correlations with time spent on recorded e-lecture study in the 2019/1 and 2019/2 classes. These 
results were in agreement with previous studies showing that students with lower academic outcomes spent a significantly higher amount of time on recorded-e-lecture study (Liles et al., 2018). While students spent a comparable amount of time in study review, students who spent their time with more recorded-e-lecture study review but with less non-recorded-e-lecture study review got worse GPA. This might be because students who used more time spent on recorded-e-lecture might be students who were not able to concentrate during class, leading to more time spent on recorded-e-lectures review to keep-up with the learning content. This could be reflective of their poor SRL in 2 aspects, which are time management and poor attention, defined as the inability to focus and concentrate during class.

Other than time management, learning strategies might also affect academic performance. Students who used less time on recorded-e-lecture review had higher GPA despite spending the same total amount of time for study review. This might also reflect that high academic achieved students had the ability to choose learning strategies which could probably be more effective in studying. Recorded-e-lecture review is a form of repeated lectures and is considered as passive learning. On the other hand, other means of lesson review, including acquiring additional materials and practicing learning exercises which are considered as active learning, could promote deeper understanding (Kusurkar, Croiset, Galindo-Garre, \& Ten Cate, 2013; Kusurkar, Ten Cate, Vos, Westers, \& Croiset, 2013). More in-depth investigation should be done on how high GPA students spend their time on study review differently from other students to better reveal the learning strategies involved in higher performance.

Students in all classes spent a similar amount of time for academic-internet use; however, time spent on nonacademic internet use was significantly lower in the Q4 group than the Q1, Q2, and Q3 groups in the 2019/2 class; and than the Q1 and Q3 groups in the 2018/2 class. Furthermore, a significant negative correlation was observed between GPA and time spent on non-academic internet use in the 2018/2 class. These results were in agreement with previous studies showing that academic performance was significantly negatively correlated with nonacademic internet use (Azizi et al., 2019; Lertwilaiwittaya et al., 2019; Sitticharoon et al., 2014; Zureick, BurkRafel, Purkiss, \& Hortsch, 2018). Too much non-academic internet use are distractors in learning, leading to poor academic performance (Zureick et al., 2018). High amount of non-academic internet use is reflective of poor time management and high levels of procrastination, which is the process of delaying the planned course of action despite the possibility of worse outcomes (Sitzmann \& Ely, 2011), and poor self-control (J. Kim, Hong, Lee, \& Hyun, 2017).

Instances of lateness and absence were significantly lowest in the Q4 group of the 2019/2 class, and were significantly highest in the Q1 group of the 2018/2 class. In addition, GPA had negative correlations with instances of lateness and absence in the 2018/2 class. These results were in accordance with previous studies showing that lower instances of absence (Liles et al., 2018; Sitticharoon et al., 2014) and lateness (Sitticharoon et al., 2014) were associated with better academic performance. However, these results were not consistent with other studies showing that class attendance had no association with academic performance (Eisen et al., 2015; Kauffman et al., 2018). This discrepancy might be due to different data collection processes since we performed fingerprint scans every morning only at the beginning of the $1^{\text {st }}$ period while the other studies recorded attendance from every period (Eisen et al., 2015; Kauffman et al., 2018). Students who are unable to arrive in class on time in the morning reflects poor self-discipline, lower effort regulation, and poor time management.

From the results regarding time spent for study review, time spent for recorded- and non-recorded-e-lecture study review, time spent for internet for academic and non-academic use, and instances of lateness and absence, it can be suggested that effective time management during both lectures and study review as well as the ability to arrive to class on time are factors contributing to good academic performance.

For motivation to study medicine, we found that students with low motivation had significantly lower GPA than students with medium or high motivation in the $1^{\text {st }}$ and $2^{\text {nd }}$ preclinical years in the 2018 class. Our results were consistent with previous studies showing that low motivation students had lower academic performance than higher motivation students (Lertwilaiwittaya et al., 2019; Pinyopornpanish et al., 2004; Sitticharoon et al., 2014). Motivation is another aspect of regulatory mechanism of SRL (Sitzmann \& Ely, 2011). Motivation reflects students' willingness to learn (Sitzmann \& Ely, 2011), which is associated with cognitive self-strategies, selfregulation (Turan, Demirel, \& Sayek, 2009), and higher effort (Kusurkar, Croiset, et al., 2013; Kusurkar, Ten Cate, et al., 2013). According to previous studies, the decrease in academic performance could be because lower motivation students had lower study effort and utilized surface study strategies (memorization) rather than deep study strategies (deep understanding) (Kusurkar, Croiset, et al., 2013; Kusurkar, Ten Cate, et al., 2013).

For happiness of students, it was significantly lowest in the Q1 group compared to the Q2, Q3, and Q4 groups in the 2018/2 class and was significantly lower in the Q1 group compared to the Q4 group in the 2019/1 class. From 
multivariate regression analysis, happiness was one of the factors that could be used to predict GPA in both the 2019 and 2018 classes. These results paralleled with previous studies showing that happiness, which is an emotion, had a positive influence on academic performance (Chen \& Lu, 2009; Datu et al., 2017). Emotion control is also a part of regulatory mechanisms of SRL. Students with better emotion control can limit intrusive thoughts, therefore reducing distraction while studying (Sitzmann \& Ely, 2011).

Stress level was comparable between groups of quartiles of students' GPA, indicating that different academic outcome levels did not necessarily lead to different stress levels. Our results parallel with a previous study showing that stress level during a regular study period did not correlate with academic achievement (Lertwilaiwittaya et al., 2019), while stress level during the period approaching the examination did (Lertwilaiwittaya et al., 2019). Academic sources of stress among medical students could be from getting low scores, a large amount of study content, taking tests and examinations, having problems in understanding the content, and not being able to follow the study schedule (Sonia Ijaz, 2017). As such, the similarity in stress level between different quartiles of GPA could be because academic stressors of medical students are not only from academic outcomes, but also from other factors as discussed above.

For prior academic achievement, we found that the students' GPA of the previous years was significantly positively associated with GPA of the current year in all 3 classes. In multivariate regression analysis, the GPA of the previous years was one of the factors that could be used to predict the GPA of the current year. These results were in agreement with a previous study showing that the previous year's GPA could predict the current year academic outcomes (Sitticharoon et al., 2014). The previous year GPA could reflect self-efficacy, which is the belief of the students' capability for success (Sitzmann \& Ely, 2011). Self-efficacy is a part of regulatory appraisal of SRL, which is the process of evaluating one's ability compared to the set goal, as well as determining which factors contribute to or prevent target achievement (Sitzmann \& Ely, 2011). Individuals with more self-efficacy will set higher goals and consequently use more effort to reach their desired goals (Sitzmann \& Ely, 2011). Other than self-efficacy, students with higher previous GPA could also reflect better developed learning skills compared to their peers (Nabizadeh, Hajian, Sheikhan, \& Rafiei, 2019). Furthermore, prior high academic achievement could also be reflective of more prior knowledge, which is an important factor influencing students' academic achievement. Taken together, the high prior academic outcome might influence the ability to acquire new knowledge as well as the integration between prior knowledge and new information (Hailikari, Katajavuori, \& Lindblom-Ylanne, 2008).

For the physical factor, BMI was associated with academic achievement. We found that obese students had significantly lower GPA than normal weight students of the 2019/1 class, and obese and overweight students had significantly lower GPA than normal weight and underweight students of the 2019/2 class. Furthermore, BMI had a significantly negative correlation with GPA in students of the 2019/2 class, and tended to have a negative correlation in students of the $2018 / 2$ class $(\mathrm{p}=0.078$ ). Our results were consistent with previous studies showing that obese students had lower GPA than non-obese students (Al-Drees et al., 2016; Suraya et al., 2017). Previous studies have shown that obesity was associated with poor self-control (Elfhag \& Morey, 2008; Gerlach, Herpertz, $\&$ Loeber, 2015). As such, the habits from low self-control could probably lead to poor self-regulation in other areas, including study habits resulting in poor academic outcomes.

In summary, factors that had positive influences on academic outcomes included higher achievement of study targets, examination expectation, time spent on non-recorded-e-lecture study, motivation to study medicine, happiness level, and previous year GPA; but lower time spent on recorded-e-lecture review, time spent on nonacademic internet use, instances of lateness to class and absence from class, and BMI. Collectively, students with more self-regulation strategies in many aspects of SRL, including time management, learning strategies, emotion control, motivation, and self-efficacy, had better academic outcomes. Enhancement of SRL in students through these strategies could help students achieve a better academic performance.

\section{Acknowledgements}

The current study was supported by the Faculty of Medicine Siriraj Hospital Medical Education Research Fund. CS is supported by the "Chalermphrakiat" Grant, Faculty of Medicine Siriraj Hospital, Mahidol University.

\section{Conflict of Interest}

All Authors declare that he/she has no conflict of interest.

\section{References}

Al-Drees, A., Abdulghani, H., Irshad, M., Baqays, A. A., Al-Zhrani, A. A., Alshammari, S. A., \& Alturki, N. I. (2016). Physical activity and academic achievement among the medical students: A cross-sectional study. 
Med Teach, 38 Suppl 1, S66-72. https://doi.org/10.3109/0142159x.2016.1142516

AlFaris, E., Irfan, F., Ponnamperuma, G., Jamal, A., Van der Vleuten, C., Al Maflehi, N., . . Ahmed, A. M. A. (2018). The pattern of social media use and its association with academic performance among medical students. Med Teach, 40(sup1), S77-s82. https://doi.org/10.1080/0142159x.2018.1465536

Appropriate body-mass index for Asian populations and its implications for policy and intervention strategies. (2004). Lancet, 363(9403), 157-163. https://doi.org/10.1016/S0140-6736(03)15268-3

Artino, A. R. J., Dong, T., DeZee, K. J., Gilliland, W. R., Waechter, D. M., Cruess, D., \& Durning, S. J. (2012). Achievement Goal Structures and Self-Regulated Learning: Relationships and Changes in Medical School. Academic Medicine, 87(10), 1375-1381. https://doi.org/10.1097/ACM.0b013e3182676b55

Azizi, S. M., Soroush, A., \& Khatony, A. (2019). The relationship between social networking addiction and academic performance in Iranian students of medical sciences: a cross-sectional study. BMC Psychol, 7(1), 28. https://doi.org/10.1186/s40359-019-0305-0

Chen, S. Y., \& Lu, L. (2009). Academic correlates of Taiwanese senior high school students' happiness. Adolescence, 44(176), 979-992.

Chung, Q. E., Abdulrahman, S. A., Khan, M. K. J., Sathik, H. B. J., \& Rashid, A. (2018). The Relationship between Levels of Physical Activity and Academic Achievement among Medical and Health Sciences Students at Cyberjaya University College of Medical Sciences. Malays $J$ Med Sci, 25(5), 88-102. https://doi.org/10.21315/mjms2018.25.5.9

Cleary, T. J., \& Sandars, J. (2011). Assessing self-regulatory processes during clinical skill performance: A pilot study. Medical Teacher, 33(7), e368-e374. https://doi.org/10.3109/0142159X.2011.577464

Datu, J. A. D., Valdez, J. P., Cabrera, I. K., \& Salanga, M. G. (2017). Subjective Happiness Optimizes Educational Outcomes: Evidence from Filipino High School Students. Span $J$ Psychol, 20, E60. https://doi.org/10.1017/sjp.2017.55

Eisen, D. B., Schupp, C. W., Isseroff, R. R., Ibrahimi, O. A., Ledo, L., \& Armstrong, A. W. (2015). Does class attendance matter? Results from a second-year medical school dermatology cohort study. Int J Dermatol, 54(7), 807-816. https://doi.org/10.1111/ijd.12816

Elfhag, K., \& Morey, L. C. (2008). Personality traits and eating behavior in the obese: poor self-control in emotional and external eating but personality assets in restrained eating. Eat Behav, 9(3), 285-293. https://doi.org/10.1016/j.eatbeh.2007.10.003

Elliot, A. J., McGregor, H. A., \& Gable, S. (1999). Achievement goals, study strategies, and exam performance: A mediational analysis. Journal of Educational Psychology, 91(3), 549-563. https://doi.org/10.1037/00220663.91.3.549

Frischenschlager, O., Haidinger, G., \& Mitterauer, L. (2005). Factors associated with academic success at Vienna Medical School: prospective survey. Croat Med J, 46(1), 58-65.

Gerlach, G., Herpertz, S., \& Loeber, S. (2015). Personality traits and obesity: A systematic review. Obes Rev, 16(1), 32-63. https://doi.org/10.1111/obr.12235

Hailikari, T., Katajavuori, N., \& Lindblom-Ylanne, S. (2008). The relevance of prior knowledge in learning and instructional design. American Journal of Pharmaceutical Education, 72(5), 113-113. https://doi.org/10.5688/aj7205113

Hill, M. R., Goicochea, S., \& Merlo, L. J. (2018). In their own words: stressors facing medical students in the millennial generation. Medical Education Online, 23(1), 1530558-1530558. https://doi.org/10.1080/10872981.2018.1530558

Kauffman, C. A., Derazin, M., Asmar, A., \& Kibble, J. D. (2018). Relationship between classroom attendance and examination performance in a second-year medical pathophysiology class. Adv Physiol Educ, 42(4), 593-598. https://doi.org/10.1152/advan.00123.2018

Kim, J., Hong, H., Lee, J., \& Hyun, M. H. (2017). Effects of time perspective and self-control on procrastination and Internet addiction. J Behav Addict, 6(2), 229-236. https://doi.org/10.1556/2006.6.2017.017

Kim, S. Y., Kim, M. S., Park, B., Kim, J. H., \& Choi, H. G. (2017). The associations between internet use time and school performance among Korean adolescents differ according to the purpose of internet use. PLoS One, 12(4), e0174878. https://doi.org/10.1371/journal.pone.0174878 
Kötter, T., Wagner, J., Brüheim, L., \& Voltmer, E. (2017). Perceived Medical School stress of undergraduate medical students predicts academic performance: an observational study. BMC Medical Education, 17(1), 256. https://doi.org/10.1186/s12909-017-1091-0

Kusurkar, R. A., Croiset, G., Galindo-Garre, F., \& Ten Cate, O. (2013). Motivational profiles of medical students: association with study effort, academic performance and exhaustion. BMC Med Educ, 13, 87. https://doi.org/10.1186/1472-6920-13-87

Kusurkar, R. A., Ten Cate, T. J., van Asperen, M., \& Croiset, G. (2011). Motivation as an independent and a dependent variable in medical education: a review of the literature. Med Teach, 33(5), e242-262. https://doi.org/10.3109/0142159x.2011.558539

Kusurkar, R. A., Ten Cate, T. J., Vos, C. M. P., Westers, P., \& Croiset, G. (2013). How motivation affects academic performance: a structural equation modelling analysis. Advances in Health Sciences Education, 18(1), 57-69. https://doi.org/10.1007/s10459-012-9354-3

Lertwilaiwittaya, P., Sitticharoon, C., Maikaew, P., \& Keadkraichaiwat, I. (2019). Factors influencing the National License Examination step 1 score in preclinical medical students. Advances in Physiology Education, 43(3), 306-316. https://doi.org/10.1152/advan.00197.2018

Liles, J., Vuk, J., \& Tariq, S. (2018). Study Habits of Medical Students: An Analysis of which Study Habits Most Contribute to Success in the Preclinical Years (Vol. 7).

Lumley, S., Ward, P., Roberts, L., \& Mann, J. P. (2015). Self-reported extracurricular activity, academic success, and quality of life in UK medical students. Int JMed Educ, 6, 111-117. https://doi.org/10.5116/ijme.55f8.5f04

Nabizadeh, S., Hajian, S., Sheikhan, Z., \& Rafiei, F. (2019). Prediction of academic achievement based on learning strategies and outcome expectations among medical students. BMC Med Educ, 19(1), 99. https://doi.org/10.1186/s12909-019-1527-9

Pinyopornpanish, M., Sribanditmongkok, P., Boonyanaruthee, V., Chan-ob, T., Maneetorn, N., \& Uuphanthasath, R. (2004). Factors affecting low academic achievement of medical students in the Faculty of Medicine, Chiang Mai University. Chiang Mai Med Bull, 43(1), 15-23. Retrieved from http://www.med.cmu.ac.th/journal/fulltext/Manee.pdf

Rabito-Alcon, M. F., \& Rodriguez-Molina, J. M. (2016). Satisfaction with life and psychological well-being in people with gender dysphoria. Actas Esp Psiquiatr, 44(2), 47-54.

Sitticharoon, C., Srisuma, S., Kanavitoon, S., \& Summachiwakij, S. (2014). Exploratory study of factors related to educational scores of first preclinical year medical students. Adv Physiol Educ, 38(1), 25-33. https://doi.org/10.1152/advan.00162.2012

Sitzmann, T., \& Ely, K. (2011). A meta-analysis of self-regulated learning in work-related training and educational attainment: What we know and where we need to go. Psychological Bulletin, 137(3), 421-442. https://doi.org/10.1037/a0022777

Sobral, D. T. (2001). An appraisal of medical students' reflection-in-learning. Medical Education, 34(3), 182-187. https://doi.org/10.1046/j.1365-2923.2000.00473.x

Sohail, N. (2013). Stress and academic performance among medical students. J Coll Physicians Surg Pak, 23(1), 67-71. https://doi.org/10.2013/jcpsp.6771

Song, H. S., Kalet, A. L., \& Plass, J. L. (2011). Assessing medical students' self-regulation as aptitude in computerbased learning. Advances in Health Sciences Education, 16(1), 97-107. https://doi.org/10.1007/s10459-0109248-1

Sonia Ijaz, H. (2017). - 7(-), - 1-4. Retrieved from - https://www.omicsonline.org/open-access/effect-of-stress-onacademic-performance-of-undergraduate-medical-students-2161-0711-1000566-95704.html?aid=95704

Stegers-Jager, K. M., Cohen-Schotanus, J., \& Themmen, A. P. N. (2012). Motivation, learning strategies, participation and medical school performance. Medical Education, 46(7), 678-688. https://doi.org/10.1111/j.1365-2923.2012.04284.x

Stewart, Lam, Betson, Wong, \& Wong (1999). A prospective analysis of stress and academic performance in the first two years of medical school. Medical Education, 33(4), 243-250. https://doi.org/10.1046/j.13652923.1999.00294.x

Suraya, F., Meo, S. A., Almubarak, Z., \& Alqaseem, Y. A. (2017). Effect of obesity on academic grades among 
Saudi female medical students at College of Medicine, King Saud University: Pilot study. J Pak Med Assoc, 67(8), 1266-1269.

Turan, S., Demirel, Ö., \& Sayek, İ. (2009). Metacognitive awareness and self-regulated learning skills of medical students in different medical curricula. Medical Teacher, 31(10), e477-e483. https://doi.org/10.3109/01421590903193521

Turan, S., \& Konan, A. (2012). Self-Regulated Learning Strategies Used in Surgical Clerkship and the Relationship with Clinical Achievement. Journal of Surgical Education, 69(2), 218-225. https://doi.org/10.1016/j.jsurg.2011.09.003

van Houten-Schat, M. A., Berkhout, J. J., van Dijk, N., Endedijk, M. D., Jaarsma, A. D. C., \& Diemers, A. D. (2018). Self-regulated learning in the clinical context: a systematic review. Medical Education, 52(10), 10081015. https://doi.org/10.1111/medu.13615

Vancouver, J. B., \& Kendall, L. N. (2006). When self-efficacy negatively relates to motivation and performance in a learning context. Journal of Applied Psychology, 91(5), 1146-1153. https://doi.org/10.1037/00219010.91.5.1146

Zureick, A. H., Burk-Rafel, J., Purkiss, J. A., \& Hortsch, M. (2018). The interrupted learner: How distractions during live and video lectures influence learning outcomes. Anatomical Sciences Education, 11(4), 366-376. https://doi.org/10.1002/ase.1754

\section{Copyrights}

Copyright for this article is retained by the author(s), with first publication rights granted to the journal.

This is an open-access article distributed under the terms and conditions of the Creative Commons Attribution license (http://creativecommons.org/licenses/by/4.0/). 